\title{
Factores que intervienen en el proceso de mineralización de nitrógeno cuando son aplicadas enmiendas orgánicas al suelo. Una revisión
}

\section{Factors involved in the process of nitrogen mineralization when organic amendments are added to a soil. A review}

ÓSCAR IVÁN MONSALVE C. ${ }^{1,3}$

JOAN SEBASTIÁN GUTIÉRREZ D. ${ }^{1}$

WILLIAM ANDRÉS CARDONA²

Plántula de mora sembrada en suelo con enmienda orgánica.

Foto: W.A. Cardona

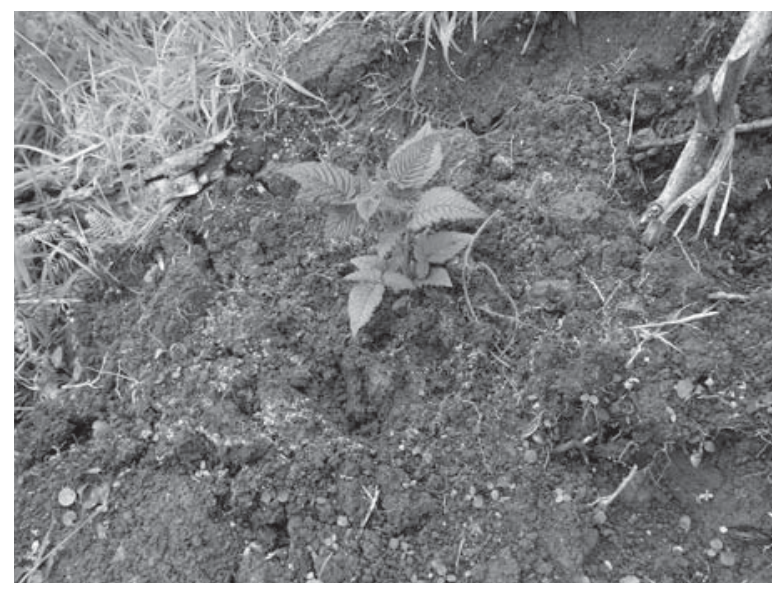

\section{RESUMEN}

La aplicación de enmiendas orgánicas es una práctica que se hace regularmente en sistemas de cultivos, con el fin de mejorar propiedades físicas y químicas del suelo y aportar nutrientes como nitrógeno $(\mathrm{N})$. Para que las plantas puedan absorberlo, gran cantidad del $\mathrm{N}$ que es aportado a través de las enmiendas orgánicas, debe ser transformado de sus formas orgánicas a inorgánicas, en un proceso denominado mineralización. Se han desarrollado investigaciones tendientes a entender este proceso, y, cómo el aporte de enmiendas orgánicas influye en su dinámica. Esta revisión proporciona una visión global sobre los factores, de suelo y enmienda, que intervienen en el proceso de mineralización de $\mathrm{N}$ cuando se aplican al suelo enmiendas orgánicas de diferentes fuentes.

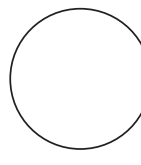

Palabras clave adicionales: inmovilización, lixiviación, abono orgánico.

\section{ABSTRACT}

The application of organic amendments is a practice that is done regularly in crop systems in order to improve the physical and chemical properties of the soil and provide nutrients such as nitrogen $(N)$. In order for plants to absorb it, much of the $\mathrm{N}$ that is contributed through organic amendments must be transformed

\footnotetext{
1 Facultad de Ciencias Agrarias, Programa de Maestría en Ciencias Agrarias, Línea Suelos y Aguas, Universidad Nacional de Colombia, Bogotá (Colombia).

2 Corporación Colombiana de Investigación Agropecuaria (Corpoica), Mosquera (Colombia).

3 Autor para correspondencia. oimonsalvec@unal.edu.co
} 
from its organic form to its inorganic form in a process called mineralization. A great amount of research has been developed to understand this process and how the contribution of organic amendments influences its dynamics. This review provides an overview of soil and amendment factors involved in the $\mathrm{N}$ mineralization process when organic amendments, from different sources, are applied to the soil.

Additional key words: immobilization, leaching, organic fertilizer.

Fecha de recepción: 09-12-2016 Aprobado para publicación: 15-04-2017

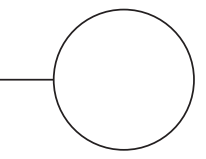

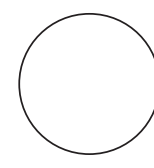

Mineralización es el proceso bioquímico mediante el cual los microorganismos del suelo, que poseen la maquinaria enzimática adecuada, obtienen la energía necesaria para realizar sus procesos metabólicos, mediante el rompimiento de los enlaces de las macromoléculas orgánicas provenientes de los residuos de las plantas, la hojarasca, la necromasa microbiana y los exudados orgánicos de las raíces, para transformarlos en moléculas inorgánicas de bajo peso molecular, que posteriormente, a través de reacciones químicas de oxidación e hidrólisis, son oxidadas catabólicamente a compuestos inorgánicos, donde estos pueden ser inmovilizados en el suelo, volatilizados al aire, lixiviados a las aguas profundas, adsorbidos en el complejo de cambio o absorbidos por las plantas y los microorganismos (p.e. Varma, 2017; Sánchez de Prager et al., 2006; Ordoñez et al., 2016).

En síntesis, y como lo exponen Azcón-Bieto y Talón (2008) y Blumenthal et al. (2003), la mineralización del $\mathrm{N}$ es la conversión de $\mathrm{N}$ orgánico en $\mathrm{N}$ inorgánico en un proceso mediado por microorganismos del suelo. La mineralización no solo se lleva a cabo en el suelo, durante el proceso de compostaje, en las enmiendas orgánicas, también ocurre el proceso de mineralización.

Enmienda orgánica es cualquier material de origen animal o vegetal que puede ser adicionado al suelo para mejorar sus propiedades físicas y/o químicas. Enmiendas como estiércoles y residuos vegetales han sido usadas para incrementar la fertilidad de los suelos desde el principio de la producción agrícola (He y Zhang, 2014). La aplicación de enmiendas orgánicas al suelo se realiza como alternativa ambientalmente favorable para el manejo de residuos orgánicos y como medio para mejorar el contenido de materia orgánica (MO) en suelos de baja fertilidad (Flavel y Murphy, 2006). Mundialmente se ha reportado un creciente interés por el uso de materiales orgánicos (Soumare et al., 2003; Petersen et al., 2004; Azeez y Van Averbeke, 2010; Mardomingo et al., 2013), debido a que estos pueden mejorar propiedades físicas, químicas y microbiológicas, e incrementar la fertilidad del suelo (Abbasi y Khizar, 2012). Además, es una manera segura y eficaz de recuperación de nutrientes como nitrógeno $(\mathrm{N})$ y fósforo $(\mathrm{P})$ para las plantas (Gilly y Eghball, 2002; Abbasi y Khizar, 2012) y de reducir el uso de fertilizantes de síntesis química para la producción de cultivos (Paterson et al., 2011).

Un importante atributo de las enmiendas orgánicas es su aporte de formas inorgánicas de $\mathrm{N}$, a corto, mediano y largo plazo. Es así, como la cantidad de $\mathrm{N}$ que retorna al suelo luego de su mineralización, contribuye con su disponibilidad para las plantas y con el crecimiento de las mismas (Abbasi y Khizar, 2012). No obstante, mucho del $\mathrm{N}$ que está contenido en las enmiendas debe ser mineralizado antes de que sea disponible para las plantas, pues este $\mathrm{N}$, en la rizosfera, continuamente recircula entre varias formas orgánicas (no disponibles) e inorgánicas (disponibles) (He y Zhang, 2014).

Cuando se realizan adiciones de enmiendas orgánicas se puede presentar la inmovilización del nitrógeno, dependiendo de la relación $\mathrm{C} / \mathrm{N}$ de los materiales aplicados, ya que compuestos con alta relación $\mathrm{C} / \mathrm{N}$ tienden a estimular la inmovilización más que la mineralización (Jenkinson, 1981). De otro lado, se puede producir pérdida de $\mathrm{N}$ a través de procesos de desnitrificación y lixiviación, generando emisión de gases de efecto invernadero (NOx) y eutroficación en los cuerpos de agua.

En esta revisión de resultados de investigación, se expone como los factores inherentes a las propiedades 
del suelo y de las enmiendas orgánicas intervienen en el proceso de mineralización de $\mathrm{N}$.

\section{FACTORES DEL SUELO}

Estudios han mostrado diferencias entre la mineralización neta de $\mathrm{N}$ para la misma enmienda en suelos de distinto origen (Thomsen y Olesen, 2000; Huang y Chen, 2009; Karyotis et al., 2006; Mubarak et al., 2010). Estas diferencias pueden ser atribuidas a propiedades como: textura (con énfasis en contenido de arcillas), capacidad de retención de humedad, $\mathrm{pH}$, salinidad, contenido de materia orgánica, concentración de nutrientes y actividad microbiana.

\section{Propiedades físicas}

Dentro de las propiedades físicas, la textura juega un importante papel en el proceso de mineralización de N del suelo. Pare y Gregorich (1999) encontraron que las enmiendas evaluadas se comportaron diferencialmente, dependiendo de la textura del suelo (arenosa, franca o arcillosa). Siendo los suelos arenosos los que mayor mineralización neta de $\mathrm{N}$ presentaron, aunque no para todas las enmiendas evaluadas. Esto puede deberse a un aumento en la aireación del suelo (Thomsen y Olesen, 2000). Matus et al. (2007) reportaron que la mineralización de $\mathrm{N}$ fluctuó entre 3,0 y $5,7 \mathrm{mg} \mathrm{kg}^{-1} \mathrm{~d}^{-1}$, siendo mayor cando la textura del suelo presentaba una mayor proporción de arcilla. Watts et al. (2007) mostraron que la mineralización del $\mathrm{N}$ orgánico en enmiendas se presentó en este orden: franco limoso $>$ franco $>$ franco arenoso. Otros autores, por otra parte, reportaron que no hubo relación entre textura y mineralización de N (Motavalli et al., 1995; González et al., 1996; Mubarak et al., 2001).

Es bien conocido que las arcillas tienen alta capacidad de retención de humedad (Delin y Linden, 2002). La influencia de la arcilla sobre el contenido de humedad del suelo y por ende sobre el contenido de oxígeno, puede afectar procesos microbianos de mineralización, inmovilización y desnitrificación (Delin y Linden, 2002).

La mineralización de $\mathrm{N}$ tiende a incrementarse cuando la humedad del suelo se aproxima a capacidad de campo y se reduce con la disminución de la humedad (Linn y Doran, 1984). No obstante, Delin y Linden (2002) encontraron en sus estudios, que la influencia del contenido de agua del suelo sobre los procesos de mineralización de $\mathrm{N}$ no son claros, debido a que los microorganismos pueden responder de manera diversa a contenidos variables de humedad. Kruse et al. (2004) encontraron que se mineralizó el $30 \%$ del $\mathrm{N}$ de la enmienda aplicada en condiciones de humedad continua del suelo, mientras que condiciones de desecación y re-humedecimiento alternado, condujeron a mínima mineralización, e incluso a inmovilización del $\mathrm{N}$.

La temperatura también juega un importante papel en la mineralización de N. Muestras de suelo incubadas a diferentes temperaturas presentaron diferencias significativas en tasas de mineralización de $\mathrm{N}\left(30^{\circ} \mathrm{C}\right.$ $>25^{\circ} \mathrm{C}>20^{\circ} \mathrm{C}$ ) (Ando et al., 1992). De igual manera, Tejada et al. (2002) encontraron que a $25^{\circ} \mathrm{C}$ la tasa de mineralización de $\mathrm{N}$ es más rápida durante los primeros días, alcanzando el pico a los 3 días de incubación, mientras que a $15^{\circ} \mathrm{C}$ el pico se alcanza a los $15 \mathrm{~d}$ de incubación, independiente del tratamiento evaluado (sin fertilizar, fertilización convencional, fertilización orgánica más convencional y fertilización organomineral). Resultados similares obtuvieron Vigil y Kissel (1995), quienes reportaron que la mineralización fue 3,7 veces más rápida a $25^{\circ} \mathrm{C}$ que a $15^{\circ} \mathrm{C}$; y 13 veces más rápida a $15^{\circ} \mathrm{C}$ que a $5^{\circ} \mathrm{C}$. La temperatura influencia notablemente la masa microbiana, pues a temperaturas mayores la proliferación de microorganismos aumenta (Tejada et al., 2002).

La densidad aparente también puede tener un importante impacto sobre la mineralización neta de $\mathrm{N}$. Torbert y Wood (1992) encontraron que la respiración del suelo (medida en términos de emisiones de $\mathrm{CO}_{2}$ ) disminuyó un $65 \%$ cuando la densidad aparente aumentó de 1,4 a 1,8 $\mathrm{g} \mathrm{cm}^{-3}$. Esto puede deberse a que en suelos más densos, con mayor contenido de arcilla, la retención de humedad es más alta, disminuyendo el contenido de aire, y a su vez el de oxígeno, provocando la disminución de los microorganismos aerobios que participan en el proceso de mineralización.

Con respecto al manejo cultural, Ringuelet y Bachmeier (2002) reportaron que hubo un 200\% más mineralización neta de $\mathrm{N}$ en suelo disturbado que en suelo no disturbado. En el trabajo realizado por Stark et al. (2007) se concluyó que la actividad enzimática, actividad microbiana y taza de mineralización de $\mathrm{N}$ es más fuertemente influenciada por factores inherentes al suelo y al ambiente, que por prácticas de manejo. Como complemento, He y Zhang (2014) indican que el C orgánico en sistemas de labranza de conservación puede estar en formas menos lábiles, lo que genera 
una disminución en las tasas de mineralización de $\mathrm{N}$. Los microorganismos requieren $\mathrm{C}$ como fuente de energía y $\mathrm{N}$ para sintetizar sus tejidos, la relación entre estos dos elementos debe permanecer dentro de cierto rango (del que se hablará más adelante) para que el proceso de mineralización se realice sin que haya inmovilización de $\mathrm{N}$ (consumo total de $\mathrm{N}$ por parte de los microorganismos) o pérdidas de $\mathrm{N}$ por volatilización o lixiviación.

\section{Propiedades químicas}

Las propiedades químicas juegan un papel fundamental en el proceso de mineralización, los microorganismos son sensibles a pHs muy ácidos o muy alcalinos, al equilibrio iónico, a la concentración de $\mathrm{C}$ y de $\mathrm{N}$ y a procesos de oxidación e hidrólisis.

Estudios han demostrado cómo la aplicación de enmiendas orgánicas puede modificar algunas propiedades químicas en los suelos. Así por ejemplo, en un suelo sódico, el pH aumentó en 0,6 puntos y se mantuvo en ese rango durante 174 d luego de la aplicación de gallinaza, mientras que con la aplicación de alfalfa, el efecto permaneció durante 56 d (Clark et al., 2007).

Clark et al. (2007) encontraron que la aplicación de enmiendas no tuvo efecto sobre la conductividad eléctrica. Al-Busaidi et al. (2014) reportaron que el origen del material orgánico influía en suelos salinos $\left(\mathrm{CE}=5,0 \mathrm{dS} \mathrm{m}^{-1}\right)$ y extremadamente salinos $(\mathrm{CE}=$ $45,1 \mathrm{dS} \mathrm{m}^{-1}$ ), donde la aplicación de residuos de pasto generó la inmovilización neta de $\mathrm{N}$, mientras que la aplicación de bovinaza resultó en tasas de mineralización de $\mathrm{N}$ positivas, para ambas condiciones de suelo.

En un estudio llevado a cabo por Delin y Linden (2002) encontraron que el contenido de materia orgánica del suelo y la mineralización de $\mathrm{N}$ presentaron una débil correlación $(r=0,26)$, lo cual contrasta con lo encontrado por Börjesson et al. (1999) y Stenberg et al. (2002), quienes encontraron una correlación más fuerte ( $r=0,69 \mathrm{y} r=0,83$, respectivamente) para las mismas variables. Por otra parte, Sano et al. (2006) encontraron que el potencial de mineralización de $\mathrm{N}$ correlacionaba significativamente con el contenido de $\mathrm{N}$ orgánico del suelo, para suelos de diferente origen.

La disponibilidad de $\mathrm{N}$ del suelo, y más aún, el contenido de $\mathrm{N}$ en su solución, es un factor determinante en la mineralización e inmovilización de $\mathrm{N}$ de las enmiendas orgánicas aplicadas (Mohanty et al., 2011). Es así, como Fangueiro et al. (2012) reportaron que una pequeña cantidad de $\mathrm{N}$ orgánico y amonio (N$\mathrm{NH}_{4}^{+}$) en la solución del suelo, mejoraba el potencial de mineralización neta de $\mathrm{N}$ de los residuos orgánicos, mientras que grandes cantidades, disminuyó el potencial de mineralización neta de N, y podría conducir a la inmovilización del mismo.

El contenido y tipo de arcilla pueden limitar la disponibilidad de $\mathrm{N}$, ya que estas son un medio de fijación de amonio (Delin y Linden, 2002). Los materiales orgánicos adicionados al suelo se mineralizan lentamente en suelos con altos contenidos de arcilla, especialmente aquellos con alta capacidad de intercambio catiónico (Thomsen et al., 2001; Mubarak et al., 2010).

Con relación al pH, Xiao et al. (2013) evaluaron el efecto de diferentes $\mathrm{pH}$ iniciales del suelo sobre el proceso de mineralización de $\mathrm{N}$, encontrando que no hubo una relación directa entre estas dos variables. Otros estudios han reportado respuestas contrarias del pH del suelo después de la incorporación de residuos de plantas: se incrementa (Marx et al., 2002; Naramabuye y Haynes, 2006; Wang et al., 2009), se disminuye (Owen et al., 1999; Rosolem, 2011), se incrementa inicialmente para luego disminuir (Paul et al., 2001; Xu y Coventry, 2003; Yuan et al., 2011) o puede permanecer invariable después de la adición de enmiendas orgánicas (Xu y Coventry, 2003).

La concentración de carbono (C) en el suelo, específicamente formas de C lábil, también determinará la mineralización de N. Keuskamp et al. (2013) encontraron que el aporte de enmiendas orgánicas promueve, además de la mineralización de $\mathrm{N}$, un incremento en la tasa en la cual el C orgánico lábil es mineralizado, mientras que disminuye la mineralización neta de C. A su vez, Zhang et al. (2015) concluyeron que la presencia de C lábil (por ejemplo en forma de glucosa) y $\mathrm{N}$ inorgánico, aumenta la tasa de mineralización de nuevos aminoácidos sintetizados a partir del aporte de enmiendas orgánicas y la mineralización de aminoácidos ya presentes en el suelo, lo cual es un mecanismo crucial en el proceso de mineralización de $\mathrm{N}$ y en el de secuestro de $\mathrm{C}$ y $\mathrm{N}$.

\section{Propiedades biológicas}

Los organismos del suelo son responsables de la transformación de $\mathrm{N}$ en formas disponibles para la planta, controlando el proceso de mineralización de N. Las bacterias del suelo son responsables de la inmovilización y mineralización de $\mathrm{N}$ del suelo y de los procesos que controlan el suministro de amonio $\left(\mathrm{NH}_{4}^{+}\right)$y 
nitrato $\left(\mathrm{NO}_{3}^{-}\right)$para las plantas. Cabe recordar, que la mineralización ocurre en dos etapas: la primera, en la que los microorganismos transforman los componentes de $\mathrm{N}$ orgánico en $\mathrm{NH}_{4}{ }^{+}$, y la segunda, en la que se sintetiza nitrato $\left(\mathrm{NO}_{3}^{-}\right)$a partir del amonio. Más específicamente, los organismos autótrofos convierten el $\mathrm{N}$ orgánico en $\mathrm{NH}_{4}{ }^{+}$, a través de un proceso llamado amonificación, mientras que los organismos heterótrofos transforman el $\mathrm{NH}_{4}^{+}$a $\mathrm{NO}_{3}$, en un proceso denominado nitrificación [(1) $\mathrm{NH}_{4}^{+}+\mathrm{O}_{2}=\mathrm{NO}_{2}{ }^{-}+4 \mathrm{H}^{+}$; (2) $\mathrm{NO}_{2}^{-}+\mathrm{H}_{2} \mathrm{O}=\mathrm{NO}_{3}^{-}+2 \mathrm{H}^{+}$] (He y Zhang, 2014).

La biomasa microbiana no solo es usada como un indicador de calidad del suelo, sino que también sirve como almacenamiento (inmovilización) y fuente (mineralización) de nutrientes para los cultivos a través de procesos de inmovilización y mineralización (Abbasi et al., 2001; Shah et al., 2010; Sabahi et al., 2010).

En un estudio en el que se evaluó el efecto de la aplicación de estiércol sobre el proceso de amonificación neta en el suelo, se encontró que el origen del material influía directamente sobre esta variable, obteniendo que la gallinaza alcanzó una amonificación total de $1,11 \mathrm{mg} \mathrm{NH}_{4}^{+} / \mathrm{kg}$ de suelo, comparado con los 0,56 $\mathrm{mg} \mathrm{NH}_{4}^{+} / \mathrm{kg}$ de suelo obtenido por la bovinaza y el control (sin aporte de N) y los $0,51 \mathrm{mg} \mathrm{NH}_{4}^{+} / \mathrm{kg}$ de suelo obtenido por la caprinaza (Azeez y Van Averbeke, 2010). Los autores sugieren que la relación $\mathrm{C} / \mathrm{N}$ de las enmiendas orgánicas influye en su tasa de mineralización, ya que un aumento en esta relación indicaría que existe mayor disponibilidad de C para el desarrollo de microorganismos amonificadores que actúan en el proceso de mineralización, por ende, se consumirían el $\mathrm{N}$ disponible, inmovilizándolo y reduciendo de esta manera la mineralización neta (Azeez y Van Averbeke, 2010; Abbasi et al., 2007).

El proceso de amonificación reduce el $\mathrm{pH}$ del suelo (Huang y Chen, 2009), debido a que en la reacción se liberan protones $\left(\mathrm{H}^{+}\right)$. Huang y Chen (2009) encontraron, que el $\mathrm{pH}$ del suelo disminuía a medida que la dosis de enmienda orgánica aumentaba. Estos autores atribuían este efecto al aumento de amonificación que se da cuando se aportan enmiendas orgánicas al suelo.

\section{FACTORES DE LA ENIMIENDA ORGÁNICA}

La tasa de mineralización de $\mathrm{N}$ de las enmiendas orgánicas depende de su relación $\mathrm{C} / \mathrm{N}$ y capacidad de descomposición, origen y tratamiento de los materiales, contenido de gramínea seca, método o duración del compostaje y de las condiciones ambientales del suelo (Antil et al., 2011). Si la cantidad de N presente en los residuos orgánicos descompuestos en más alta que la requerida por los microorganismos, habrá mineralización neta con liberación de $\mathrm{N}$ inorgánico. Si la cantidad de $\mathrm{N}$ en los residuos es igual a la cantidad requerida, no habrá mineralización neta. Si, por otro lado, la cantidad de $\mathrm{N}$ presente en los residuos es más pequeña que la requerida por los microorganismos, se requerirá inmovilización de $\mathrm{N}$ inorgánico adicional, proveniente del suelo (Corbeels et al., 1999). Esto sugiere que la cantidad de $\mathrm{N}$ y $\mathrm{C}$ en las enmiendas y necromasa microbiana son factores que controlan la ocurrencia de la mineralización o inmovilización neta de N (Cabrera et al., 2005). Algunos estudios muestran que la relación $\mathrm{C} / \mathrm{N}$ que genera un punto de equilibrio entre mineralización e inmovilización neta de $\mathrm{N}$ puede estar entre 20 y 40 (Van Kessel et al., 2000; Seneviratne, 2000; Oian y Schoenau, 2002). No obstante, según Gilmour (1998), ese punto de equilibro se encuentra alrededor de 15.

Enmiendas orgánicas con similares relaciones $\mathrm{C} / \mathrm{N}$ pueden mineralizar diferentes cantidades de $\mathrm{N}$, debido a sus diferencias en composición, que no son reflejadas por esta relación (Cabrera et al., 2005). Debido a esto, se han desarrollado diversos estudios, donde no solo se evalúa la relación $\mathrm{C} / \mathrm{N}$, sino que además se determina la composición de diferentes compuestos o grupo de compuestos presentes en las enmiendas (Cabrera et al., 2005), tales como polifenoles, proteínas, carbohidratos solubles, hemicelulosa, celulosa y lignina, y encontraron que estos están relacionados con la descomposición de residuos (Thuriés et al., 2001) y mineralización neta (Vigil y Kissel, 1995; Seneviratne, 2000).

Algunos estudios han encontrado relaciones significativas entre el potencial de mineralización de $\mathrm{N}$ y la fracción hidrosoluble en los residuos orgánicos (Cabrera et al., 2005). Por ejemplo, Qafoku et al. (2001) reportaron que el potencial de mineralización de $\mathrm{N}$ se correlacionó fuertemente con el contenido de $\mathrm{N}$ orgánico hidrosoluble $(r=0,93)$ y se correlacionó débilmente con la relación C/N $(r=-0,26)$. De Neve y Hoffman (1996) obtuvieron resultados similares, encontrando una correlación de $r=0,86$ entre potencial de mineralización de $\mathrm{N}$ y contenido de $\mathrm{N}$ orgánico hidrosoluble; y una correlación de $r=-0,76$ entre potencial de mineralización de $\mathrm{N}$ y relación $\mathrm{C} / \mathrm{N}$. 
Cuando se hacen aportes de enmiendas orgánicas al suelo, la actividad microbiana, expresada en términos de respiración $\left[\mathrm{C}-\mathrm{CO}_{2}\left(\mathrm{mg} \mathrm{kg}^{-1}\right.\right.$ día $\left.\left.^{-1}\right)\right]$, se incrementa en este orden: 1) mezcla (fuente mineral + residuos vegetales + estiércol); 2) mezcla residuos vegetales + estiércol; 3) residuos vegetales; 4) mezcla (fuente mineral + estiércol); 5) estiércol; 6) fuente mineral más residuos vegetales, 7) fuente mineral y 8) sin aplicación de N (Abbasi y Khizar, 2012). De igual manera, la mineralización de $\mathrm{N}$, producto de la actividad microbiana, siguió la misma tendencia mostrada en la respiración (Abbasi y Khizar, 2012). Lo que indica que hay una relación directa entre actividad microbiana y tasa de mineralización de N. Es así, como la adición de enmiendas orgánicas resulta en un incremento en la cantidad de $\mathrm{N}$ de la biomasa microbiana, sin importar el tipo de suelo.

El comportamiento de la enmienda variará de acuerdo con su origen, ya sea de estiércol animal o de residuo vegetal, e incluso del tipo de animal, dieta, edad y sistema de manejo (Chadwick et al., 2000). Así por ejemplo, Azeez y Van Averbeke (2010) encontraron, que las relaciones $\mathrm{C} / \mathrm{N}$ y $\mathrm{N} / \mathrm{P}$ se presentaron en este orden de magnitud, de acuerdo con su origen: caprinaza $>$ bovinaza > gallinaza. Por lo tanto, es de esperarse que la gallinaza se mineralice más rápido que la bovinaza y que la caprinaza (Chadwick et al., 2000; Oiu et al., 2008). Nourbakhsh y Dick (2005) encontraron que el origen de la enmienda orgánica mostró diversas tasas de mineralización de $\mathrm{N}$, dependiendo de su concentración de celulosa, hemicelulosa, lignina y relación $\mathrm{C}: \mathrm{N}$, presentando una correlación negativa entre estos compuestos y la concentración de $\mathrm{N}$ y positiva entre la concentración de $\mathrm{N}$ y la tasa de mineralización, lo que se corrobora con los resultados presentados por Pansu y Thuriés (2003), donde el origen de la enmienda orgánica generó diferentes patrones de mineralización de $\mathrm{N}$ (inmovilización, mineralización moderada, mineralización marcada y altas tasas de transformación con posible pérdida de gases), dependiendo de su origen y propiedades químicas. Rao y Li (2003) encontraron que la mineralización de $\mathrm{N}$ en un suelo calcáreo fue mayor para las enmiendas de residuos de leguminosas que para aquellas provenientes de otros residuos vegetales. Nourbakhsh y Dick (2005), además, reportaron que la concentración de $\mathrm{N}$ de residuos vegetales utilizados como enmienda es la variable que más se correlaciona con la dinámica de mineralización de $\mathrm{N}$ en suelo $(r=0,96)$, seguido de la relación $\mathrm{C} / \mathrm{N}(r=-0,69)$, y la relación lignina/ $\mathrm{N}(r=$ $-0,68)$. Cabe anotar, que en este estudio se evaluaron un número considerable de residuos vegetales (14) de diversos orígenes, lo cual produjo que la inmovilización y mineralización neta de $\mathrm{N}$ fluctuara en un rango amplio (-59,7 a 622,2 $\mathrm{mg} \mathrm{N} / \mathrm{kg}$ de suelo).

Aoyama y Nozawa (1993) encontraron que la cantidad de $\mathrm{N}$ acumulado en la biomasa microbiana va a depender del material orgánico aplicado al suelo. Así, aplicaciones de residuos de granja resultaron en una inmovilización de $\mathrm{N}$ (acumulación de $\mathrm{N}$ en biomasa microbiana) mayor que la encontrada con la aplicación de gallinaza (13,5 y 4\%, respectivamente). Por otro lado, Abbasi et al. (2007) registraron que no hubo diferencias en la mineralización de $\mathrm{N}$ entre tres diferentes enmiendas utilizadas, pero si hubo mayor mineralización (42-43\%) con respecto al suelo sin aplicación de enmiendas. Abbasi et al. (2007) mostraron que las variaciones de la mineralización de $\mathrm{N}$ entre diferentes enmiendas orgánicas se correlacionaron fuertemente con su contenido inicial de $\mathrm{N}$, lignina, relación $\mathrm{C} / \mathrm{N}$, y relación lignina/ $\mathrm{N}$.

Las fracciones de agregados por tamaño de partícula de la enmienda también influyen sobre el proceso de mineralización de N (Kumar et al., 2002). Es así, como Doublet et al. (2010) encontraron que la fracción $0-50 \mu \mathrm{m}$ produjo la mayor mineralización de $\mathrm{N}$ de la enmienda aplicada al suelo, mientras que otras fracciones (> $200 \mu \mathrm{m}$ ) fueron más fácilmente biodegradables e indujeron la inmovilización del $\mathrm{N}$.

Castellanos y Pratt (1981) mostraron que el N disponible liberado de estiércoles compostados fue solo la mitad del $\mathrm{N}$ liberado por estiércoles no compostados. Mardomingo et al. (2013) encontraron que las mismas enmiendas orgánicas, pero provenientes de digestión aérobica y anaeróbica, produjeron diferencias en la actividad microbiana del suelo, en términos de actividad enzimática y respiración basal.

Evaluaciones con enmiendas orgánicas tipo biochar, muestran que su aplicación no produce efecto significativo sobre la mineralización neta de $\mathrm{N}$, debido a que aumenta la capacidad de retención de $\mathrm{N}\left(\mathrm{NH}_{4}{ }^{+}\right.$ y $\mathrm{NO}_{3}^{-}$) (Ding et al., 2010; Zhao et al., 2013) y la capacidad de retención de C estable (Lehmann, 2007; Woolf et al., 2010), lo que conlleva a la disminución de la actividad microbiana del suelo (Kim et al., 2015).

\section{CONCLUSIONES}

El estudio del proceso de mineralización de $\mathrm{N}$ cuando son aplicadas enmiendas orgánicas al suelo es amplio 
y contrastante. Se puede evidenciar que la mineralización neta de $\mathrm{N}$, así como su tasa de mineralización, dependen, tanto de las propiedades físicas, químicas y microbiológicas del suelo, como de la composición y origen de la enmienda orgánica y las condiciones ambientales donde se desarrolla el proceso. Esto ha llevado a que sea muy difícil establecer relaciones directas entre la dinámica del proceso de mineralización e inmovilización de $\mathrm{N}$ en un suelo dado, y la composición física, química y biológica de la enmienda orgánica.

No obstante, se tiene una cantidad de información que ayudaría a dilucidar, en cierta medida, cómo sería la dinámica del $\mathrm{N}$ en el suelo cuando se conocen sus propiedades y composición y además se tiene bien caracterizada la enmienda orgánica a aplicar.

\section{REFERENCIAS BIBLIOGRÁFICAS}

Abbasi, M.K. y A. Khizar. 2012. Microbial biomass carbon and nitrogen transformations in a loam soil amended with organic-inorganic $\mathrm{N}$ sources and their effect on growth and N-uptake in maize. Ecol. Eng. 39, 123-132. Doi: 10.1016/j.ecoleng.2011.12.027

Abbasi, M.K., M. Hina, A. Khalique y S.R. Khan. 2007. Mineralization of three organic manures used as nitrogen source in a soil incubated under laboratory conditions. Comm. Soil Sci. Plant Anal. 38, 1691-1711. Doi: 10.1080/00103620701435464

Abbasi, M.K., Z. Shah y W.A. Adams. 2001. Mineralization and nitrification potentials of grassland soils at shallow depth during laboratory incubation. J. Plant Nutr. Soil Sci. 164, 407-502. Doi: 10.1002/1522-2624(200110)164:5<497::AID-JPLN497>3.0.CO;2-E

Al-Busaidi, K.T.S., A. Buerkert y R.G. Joergensen. 2014. Carbon and nitrogen mineralization at different salinity levels in Omani low organic matter soils. J. Arid Environ. 100-101, 106-110. Doi: 10.1016/j. jaridenv.2013.10.013

Ando, H., R.C. Aragones y G. Wada. 1992. Mineralization pattern of soil organic $\mathrm{N}$ of several soils in the tropics. Soil Sci. Plant Nutr. 38, 227-234. Doi: 10.1080/00380768.1992.10416485

Antil, R., A. Bar-Tal, P. Fine y A. Hadas. 2011. predicting nitrogen and carbon mineralization of composted manure and sewage sludge in soil. Compost Sci. Util. 19, 33-43. Doi: 10.1080/1065657X.2011.10736974

Aoyama, M. y T. Nozawa. 1993. Microbial biomass nitrogen and mineralization-immobilization processes of nitrogen in soils incubated with various organic materials. Soil Sci. Plant Nutr. 39, 23-32. Doi: 10.1080/00380768.1993.10416971
Azcón-Bieto, J. y M. Talón.2008. Fundamentos de fisiología vegetal. $2^{a}$ ed. McGraw-Hill - Interamericana y UBE Editores, Madrid, España.

Azeez, J.O. y W. Van Averbeke. 2010. Nitrogen mineralization potential of three animal manures applied on a sandy clay loam soil. Biores. Technol. 101(14), 56455651. Doi: 10.1016/j.biortech.2010.01.119

Blumenthal, D.M., N.R. Jordany M.P. Russelle. 2003. Soil carbon addition controls weeds and facilitates prairie restoration. Ecol. Appl. 13, 605-615. Doi: 10.1890/1051-0761(2003)013 [0605:SCACWA]2.0. $\mathrm{CO} ; 2$

Börjesson, T., B. Stenberg, B. Lindén y A. Jonsson. 1999. NIR spectroscopy, mineral nitrogen analysis and soil incubations for the prediction of crop uptake of nitrogen during the growing season. Plant Soil 214, 75-83. Doi: 10.1023/A:1004775524189

Cabrera, M.L., D.E. Kissel y M.F. Vigil. 2005. Nitrogen mineralization from organic residues: research opportunities. J. Environ. Qual. 34, 75-79. Doi: 10.2134/ jeq2005.0075

Castellanos, J.Z. y P.F. Pratt. 1981. Mineralization of manure nitrogen - correlation with laboratory indexes. Soil Sci. Soc. Amer. J. 45, 354-357. Doi: 10.2136/ sssaj1981.03615995004500020025x

Chadwick, D.R., F. John, B.F. Pain, B.J. Chambersy J.C. Williams. 2000. Plant uptake of nitrogen from the organic nitrogen fraction of animal manures: a laboratory experiment. J. Agric. Sci. 134, 159-168. Doi: 10.1017/ S0021859699007510

Clark, G.J., N. Dodgshun, P.W.G. Sale y C. Tang. 2007. Changes in chemical and biological properties of a sodic clay subsoil with addition of organic amendments. Soil Biol. Biochem. 39, 2806-2817. Doi: 10.1016/j. soilbio.2007.06.003

Corbeels, M., G. Hofmany O. Van Cleemput. 1999. Simulation of net $\mathrm{N}$ immobilization and mineralisation in substrate-amended soils by the NCSOIL computer model. Biol. Fertil. Soils 28, 422-430. Doi: 10.1007/ s003740050515

De Neve, S. y G. Hofman. 1996. Modelling N mineralization of vegetable crop residues during laboratory incubations. Soil Biol. Biochem. 28, 1451-1457. Doi: 10.1016/S0038-0717(96)00154-X

Delin, S. y B. Lindén. 2002. Relations between net nitrogen mineralization and soil characteristics within an arable field. Acta Agric. Scand. Sect. B Soil Plant Sci. 52, 78-85. Doi: 10.1080/090647102321089819

Ding, Y., Y. Liu, W. Wu, D. Shi, M. Yang y Z. Zhong. 2010. Evaluation of biochar effects on nitrogen retention and leaching in multi-layered soil columns. Water Air Soil Poll. 213, 47-55. Doi: 10.1007/s11270-010-0366-4

Doublet, J., C. Francou, J.P. Pétraud, M.F. Dignac, M. Poitrenaud y S. Houot. 2010. Distribution of C and N 
mineralization of a sludge compost within particle-size fractions. Bioresource Technol. 101(4), 1254-1262. Doi: 10.1016/j.biortech.2009.09.037

Fangueiro, D., G. de Sousa, E. Vasconcelos y E. Duarte. 2012. Influence of nitrogen content in the soil solution on potential nitrogen mineralization of organic residues. Arch. Agron. Soil Sci. 58, S112-S115. Doi: 10.1080/03650340.2012.694136

Flavel, T.C. y D.V. Murphy. 2006. Carbon and nitrogen mineralization rates after application of organic amendments to soil. J. Environ. Qual. 35, 183. Doi: 10.2134/ jeq2005.0022

Gilly, J.E. y B. Eghball. 2002. Residual effects of compost and fertilizer applications on nutrients in runoff. Trans. Amer. Soc. Agric. Biol. Eng. 45, 1905-1910.

Gilmour, J.T. 1998. Carbon and nitrogen mineralization during co- utilization of biosolids and composts. pp. 89-112. En: Brown, S., J.S. Angle y L. Jacobs (eds.). Beneficial co-utilization of agricultural, municipal, and industrial by-products. Kluwer Academic Publ., Dordrecht, The Netherlands. Doi: 10.1007/978-94-011-5068-2_8

González, P.S.J., A. Cabaneiro, M.C. Villar, M. Carballas y T. Carballa. 1996. Effect of soil characteristics on $\mathrm{N}$ mineralization capacity in 112 native and agricultural soils from the northwest of Spain. Biol. Fert. Soils 22, 252-260. Doi: 10.1007/BF00382521

He, Z. y H. Zhang (eds.). 2014. Applied manure and nutrient chemistry for sustainable agriculture and environment. Springer, New York, USA. Doi: 10.1007/978-94-017-8807-6

Huang, C.C. y Z.S. Chen. 2009. Carbon and nitrogen mineralization of sewage sludge compost in soils with a different initial pH. Soil Sci. Plant Nutr. 55, 715-724. Doi: 10.1111/j.1747-0765.2009.00410

Jenkinson, D.S. 1981. The fate of plant and animal residues in soil. pp. 505-561. En: Greenland, D.J. y M.H.B. Hayes (eds.). The chemistry of soil processes. John Wiley \& Sons, New York, USA.

Mardomingo, J.I., R.P. Soler, M.Á. Casermeiro, M.T. de la Cruz y A. Polo. 2013. Seasonal changes in microbial activity in a semiarid soil after application of a high dose of different organic amendments. Geoderma 206, 40-48. Doi: 10.1016/j.geoderma.2013.04.025

Karyotis, T., t. Mitsimponas, M. Tziouvalekas y C. Noulas. 2006. Net nitrogen and sulfur mineralization in mountainous soils amended with indigenous plant residues. Comm. Soil Sci. Plant Anal. 37, 2805-2817. Doi: 10.1080/00103620600832605

Keuskamp, J.A., H. Schmitt, H.J. Laanbroek, J.T.A. Verhoeven y M.M. Hefting. 2013. Nutrient amendment does not increase mineralisation of sequestered carbon during incubation of a nitrogen limited mangrove soil. Soil Biol. Biochem. 57, 822-829. Doi: 10.1016/j. soilbio.2012.08.007

Kim, T.T.C., M.T. Rose, T.R. Cavagnaroy A.F. Patti. 2015. Lignite amendment has limited impacts on soil microbial communities and mineral nitrogen availability. Appl. Soil Ecol. 95, 140-150. 10.1016/j.apsoil.2015.06.020

Kruse, J., D.E. Kissel y M.L. Cabrera. 2004. Effects of drying and rewetting on carbon and nitrogen mineralization in soils and incorporated residues. Nutr. Cycl. Agroecosyst. 69, 247-256. Doi: 10.1023/B:FRES.0000035197.57441.cd

Kumar, K., C.J. Rosen y S.C. Gupta. 2002. Kinetics of nitrogen mineralization in soils amended with sugar beet processing by-products. Comm. Soil Sci. Plant Anal. 33, 3635-3651. Doi: 10.1081/CSS-120015912

Lehmann, J. 2007. A handful of carbon. Nature 447, 143144. Doi: $10.1038 / 447143 a$

Linn D. M. y J.W. Doran. 1984. Effect of water-filled pore space on carbon dioxide and nitrous oxide production in tilled and notilled soils. Soil Sci. Soc. Amer. J. 48,1267-1272. Doi: 10.2136/ sssaj1984.03615995004800060013x

Marx, M., B. Marschner y P. Nelson, P. 2002. Short-term effects of incubated legume and grass materials on soil acidity and $\mathrm{C}$ and $\mathrm{N}$ mineralization in a soil of northeast Australia. Aust. J. Soil Resp. 40, 1231-1241. Doi: 10.1071/SR01099

Matus, F.J., C.H. Lusk y C.R. Maire. 2007. Effects of soil texture, carbon input rates, and litter quality on free organic matter and nitrogen mineralization in Chilean rain forest and agricultural soils. Comm. Soil Sci. Plant Anal. 39, 187-201. Doi: 10.1080/00103620701759137

Mohanty, M., K.S. Reddy, M.E. Probert, R.C. Dalal, A.S. Rao y N.W. Menzies. 2011. Modelling N mineralization from green manure and farmyard manure from a laboratory incubation study. Ecol. Model. 222(3), 719726. Doi: 10.1016/j.ecolmodel.2010.10.027

Motavalli, P.P., C.A. Palm, E.T. Elliott, S.D. Frey y P.C. Smithson. 1995. Nitrogen mineralization in humid tropical forest soils: Mineralogy, texture, and measured nitrogen fractions. Soil Sci. Soc. Amer. J. 59, 1168-1175. Doi: 10.2136/sssaj1995.03615995005900040032x

Mubarak, A.R., A.B. Rosenani, S.D. Zauyah y A.R. Anuar. 2001. Nitrogen mineralization from crop residues in selected tropical soils. Trop. Agric. 78,165-173.

Mubarak, A.R., E.A.M. Gali, A.G. Mohamed, D. Steffens y A.H. Awadelkarim. 2010. Nitrogen mineralization from five manures as influenced by chemical composition and soil type. Comm. Soil Sci. Plant Anal. 41, 1903-1920. Doi: 10.1080/00103624.2010.495802

Naramabuye, F.X. y R.J. Haynes. 2006. Effect of organic amendments on soil $\mathrm{pH}$ and $\mathrm{Al}$ solubility and use of laboratory indices to predict their liming effect. Soil Sci. 171, 754-763. Doi: 10.1097/01.ss.0000228366.17459.19 
Nourbakhsh, F. y R.P. Dick. 2005. Net nitrogen mineralization or immobilization potential in a residue-amended calcareous soil. Arid Land Res. Manag. 19, 299-306. Doi: 10.1080/15324980500299615

Ordoñez, Y.M., B.R. Fernandez, L.S. Lara, A. Rodriguez, D. Uribe y I. R. Sanders. 2016. Bacteria with phosphate solubilizing capacity alter mycorrhizal fungal growth both inside and outside the root and in the presence of native microbial communities. PLoS ONE 11(6), e0154438. Doi: 10.1371/journal.pone.0154438

Owen, K.M., R.H. Marrs, C.S.R. Snow y C.E. Evans. 1999. Soil acidification e the use of sulphur and acidic plant materials to acidify arable soils for the recreation of heathland and acidic grassland at Minsmere. Biol. Conserv. 87, 105-121. Doi: 10.1016/ S0006-3207(98)00027-5

Pansu, M. y L. Thuriès. 2003. Kinetics of $C$ and $N$ mineralization, $\mathrm{N}$ immobilization and $\mathrm{N}$ volatilization of organic inputs in soil. Soil Biol. Biochem 35, 37-48. Doi: 10.1016/S0038-0717(02)00234-1

Pare, T. y E.G. Gregorich. 1999. Soil textural effects on mineralization of nitrogen from crop residues and the added nitrogen interaction. Comm. Soil Sci. Plant Anal. 30, 145-157. Doi: 10.1080/00103629909370191

Paterson, E., R. Neilson, A.J. Midwood, S.M. Osborne, A. Sim, B. Thornton y P. Millard. 2011. Altered food web structure and C-flux pathways associated with mineralisation of organic amendments to agricultural soil. Appl. Soil Ecol. 48(2), 107-116. Doi: 10.1016/j. apsoil.2011.04.006

Paul, K.I., A.S. Black y M.K. Conyers. 2001. Influence of fallow, wheat and subterranean clover on $\mathrm{pH}$ within an initially mixed surface soil in the field. Biol. Fertility Soils 33, 41-52. Doi: 10.1007/s003740000288

Petersen, S.L., B.A. Roundy y R.M. Bryant. 2004. Revegetation methods for high elevation roadsides at Bryce Canyon National Park, Utah. Restoration Ecol. 12, 248-257. Doi: 10.1111/j.1061-2971.2004.00321.x

Qafoku, O.S., M.L. Cabrera, W.R. Windham y N.S. Hill. 2001. Rapid methods to determine potentially mineralizable nitrogen in broiler litter. J. Environ. Qual. 30, 217-221. Doi: 10.2134/jeq2001.301217x

Qian, P. y J. Schoenau. 2002. Availability of nitrogen in solid manure amendments with different C:N ratios. Can. J. Soil Sci. 82, 219-225. Doi: 10.4141/S01-018

Qiu, S., A.J. McComb y R.W. Bell 2008. Ratios of C, N and P in soil water direct microbial immobilisation-mineralization and $\mathrm{N}$ availability in nutrient amended sandy soils in southwestern Australia. Agric. Ecosyst. Environ. 127, 93-99. Doi: 10.1016/j.agee.2008.03.002

Rao, R.B. y Y.C. Li. 2003. Nitrogen mineralization of cover crop residues in calcareous gravelly soil. Comm. Soil Sci. Plant Anal. 34, 299-313. Doi: 10.1081/ css-120017822
Ringuelet, A. y O.A. Bachmeier. 2002. Kinetics of soil nitrogen mineralization from undisturbed and disturbed soil. Comm. Soil Sci. Plant Anal. 33, 3703-3721. Doi: 10.1081/CSS-120015916

Rosolem, C.A. 2011. Exchangeable basic cations and nitrogen distribution in soil as affected by crop residues and nitrogen. Braz. Arch. Biol. Technol. 54, 441-450. Doi: 10.1590/S1516-89132011000300003

Sabahi, H., H. Veisi, S. Soufizadeh y K.S.Asilan, K.S. 2010. Effect of fertilization systems on soil microbial biomass and mineral nitrogen during Canola (Brassica napus L.) development stages. Comm. Soil Sci. Plant Anal. 41, 1665-1673. Doi: 10.1080/00103624.2010.489132

Sánchez de Prager, M., A. Rojas, J. Pérez, O. Zúñiga y J.M. Gascó. 2006. Actividad y biomasa microbianas como indicadores de materia orgánica en sistemas de cultivo de maracuyá (Passiflora edulis) en Toro, Valle del Cauca, Colombia. Acta Agron. 55(4), 7-12.

Sano, S., J. Yanai y T. Kosaki. 2006. Relationships between labile organic matter and nitrogen mineralization in Japanese agricultural soils with reference to land use and soil type. Soil Sci. Plant Nutr. 52, 49-60. Doi: 10.1111/j.1747-0765.2006.00003

Seneviratne, G. 2000. Litter quality and nitrogen release in tropical agriculture: A synthesis. Biol. Fertility Soils 31, 60-64. Doi: 10.1007/s003740050624

Shah, Z., R.S. Ahmad y H.U. Rahman. 2010. Soil microbial biomass and activities as influenced by green manure legumes and $\mathrm{N}$ fertilizer in rice-wheat system. Pak. J. Bot. 42, 2589-2598.

Soumare, M., F.M.G. Tack y M.G. Verloo. 2003. Effects of a municipal solid waste compost and mineral fertilization on plant growth in two tropical agricultural soils of Mali. Bioresource Technol. 86, 15-20. Doi: 10.1016/ S0960-8524(02)00133-5

Stark, C., L.M. Condron, A. Stewart, H.J. Di y M. O’Callaghan. 2007. Influence of organic and mineral amendments on microbial soil properties and processes. Appl. Soil Ecol. 35, 79-93. Doi: 10.1016/j.apsoil.2006.05.001

Stenberg, B., A. Jonsson y T. Börjesson. 2002. NIR-technology for rationale soil analysis with implications for precision agriculture. En: Near infrared spectroscopy: Changing the world with NIR. NIR Publications, Chichester.

Tejada, M., C. Benitez y J.L. Gonzalez. 2002. Nitrogen mineralization in soil with conventional and organomineral fertilization practices. Comm. Soil Sci. Plant Anal. 33, 3679-3702. Doi: 10.1081/CSS-120015915

Thomsen, I.K., J.E. Olesen, P. Schoenning, B. Jensen y B.T. Christensen. 2001. Net mineralization of soil $\mathrm{N}$ and $15 \mathrm{~N}$-ryegrass residues in differently textured soils of similar mineralogical composition. Soil Biol. Biochem. 33, 277-285. Doi: 10.1016/S0038-0717(00)00138-3 
Thomsen, I.K. y J.E. Olesen. 2000. C and N mineralization of composted and anaerobically stored ruminant manure in differently textured soils. J. Agric. Sci. 135, 151-159. Doi: 10.1017/S0021859699008096

Thuriés, L., M. Pansu, C. Feller, P. Herrmann y J.C. Rémy. 2001. Kinetics of added organic matter decomposition in a Mediterranean sandy soil. Soil Biol. Biochem 33(78), 997-1010. Doi: 10.1016/S0038-0717(01)00003-7

Torbert, H.A. y C. Wood. 1992. Effects of soil compaction and water-filled pore space on soil microbial activity and $\mathrm{N}$ losses. Comm. Soil Sci. Plant Anal. 23, 13211331. Doi: $10.1080 / 00103629209368668$

Van Kessel, J.S., J. Reeves y J. Meisinger. 2000. Nitrogen and carbon mineralization of potential manure components. J. Environ. Qual. 29, 1669-1677.

Varma, A. (ed.). 2017. Soil biology. Springer, New York, USA.

Vigil, M.F. y D.E. Kissel. 1995. Rate of nitrogen mineralized from incorporated crop residues as influenced by temperature. Soil Sci. Soc. Amer. J. 59, 1636-1644. Doi: 10.2136/sssaj1995.03615995005900060019x

Wang, N., J.Y. Li y R.K. Xu. 2009. Use of agricultural by-products to study the $\mathrm{pH}$ effects in an acid tea garden soil. Soil Use Manag. 25, 128-132. Doi: 10.1111/j.1475-2743.2009.00203.x

Watts, D.B., H. Torbert y S.A. Prior. 2007. Mineralization of nitrogen in soils amended with dairy manure as affected by wetting/drying cycles. Comm. Soil Sci. Plant Anal.38, 2103-2116. Doi: 10.1080/00103620701548860
Woolf, D., J.E. Amonette, F. Street-Perrott, J. Lehmann y S. Joseph. 2010. Sustainable biochar to mitigate global climate change. Nature Comm. 1, 56. Doi: 10.1038/ ncomms1053

Xiao, K., J. Xu, C. Tang, J. Zhang y P.C. Brookes. 2013. Differences in carbon and nitrogen mineralization in soils of differing initial $\mathrm{pH}$ induced by electrokinesis and receiving crop residue amendments. Soil Biol. Biochem. 67, 70-84. Doi: 10.1016/j.soilbio.2013.08.012

Xu, R.K. y D.R. Coventry. 2003. Soil pH changes associated with lupin and wheat plant materials incorporated in a red-brown earth soil. Plant Soil 250, 113-119. Doi: 10.1023/A:1022882408133

Yuan, J.H., R.K. Xu, W. Oian y R.H. Wang. 2011. Comparison of the ameliorating effects on an acidic ultisol between four crop straws and their biochars. J. Soils Sediments 11, 741-750. Doi: 10.1007/s11368-011-0365-0

Zhang, W., C. Liang, J. Kao-Kniffin, H. He, H. Xie, H. Zhang, H. y X. Zhang. 2015. Differentiating the mineralization dynamics of the originally present and newly synthesized amino acids in soil amended with available carbon and nitrogen substrates. Soil Biol. Biochem. 85, 162-169. Doi: 10.1016/j.soilbio.2015.03.004

Zhao, X., S. Wang y G. Xing. 2013. Nitrification, acidification, and nitrogen leaching from subtropical cropland soils as affected by rice straw-based biochar: laboratory incubation and column leaching studies. J. Soils Sediments 14(3), 471-482. Doi: 10.1007/ s11368-013-0803-2 